\title{
Lactobacillus paracasei GMNL-32, Lactobacillus reuteri GMNL-89 and L. reuteri GMNL-263 ameliorate hepatic injuries in lupus-prone mice
}

\author{
Tsai-Ching Hsu ${ }^{1,2,3}$, Chih-Yang Huang ${ }^{4,5,6}$, Chung-Hsien $\mathrm{Liu}^{7}$, Kuo-Ching $\mathrm{Hsu}^{1}$, Yi-Hsing Chen ${ }^{8,9 *}$ and \\ Bor-Show Tzang ${ }^{1,2,3,10 *}$ \\ ${ }^{1}$ Institute of Biochemistry, Microbiology and Immunology, Chung Shan Medical University, Taichung 402, Taiwan, ROC \\ ${ }^{2}$ Immunology Research Center, Chung Shan Medical University, Taichung 402, Taiwan, ROC \\ ${ }^{3}$ Clinical Laboratory, Chung Shan Medical University Hospital, Taichung 402, Taiwan, ROC \\ ${ }^{4}$ Graduate Institute of Basic Medical Science, China Medical University, Taichung 404, Taiwan, ROC \\ ${ }^{5}$ Graduate Institute of Chinese Medical Science, China Medical University, Taichung 404, Taiwan, ROC \\ ${ }^{6}$ Department of Health and Nutrition Biotechnology, Asia University, Taichung 413, Taiwan, ROC \\ ${ }^{7}$ Department of Obstetrics and Gynecology, Chung Shan Medical University and Chung Shan Medical University Hospital, \\ Taichung 402, Taiwan, ROC \\ ${ }^{8}$ Research and Development Department, GenMont Biotech Incorporation, Tainan 741, Taiwan, ROC \\ ${ }^{9}$ Institute of Biomedical Science and Rong Hsing Research Center for Translational Medicine, National Chung-Hsing \\ University, Taichung 402, Taiwan, ROC \\ ${ }^{10}$ Department of Biochemistry, School of Medicine, Chung Shan Medical University, Taichung 402, Taiwan, ROC
}

(Submitted 8 December 2016 - Final revision received 14 March 2017 -Accepted 2 April 2017-First published online 15 May 2017)

\section{Abstract}

Probiotics are known to regulate host immunity by interacting with systemic and mucosal immune cells as well as intestinal epithelial cells. Supplementation with certain probiotics has been reported to be effective against various disorders, including immune-related diseases. However, little is known about the effectiveness of Lactobacillus paracasei GMNL-32 (GMNL-32), Lactobacillus reuteri GMNL-89 (GMNL-89) and L. reuteri GMNL-263 (GMNL-263) in the management of autoimmune diseases, especially systemic lupus erythematosus (SLE). NZB/W F1 mice, which are a lupus-prone animal model, were orally gavaged with GMNL-32, GMNL-89 or GMNL-263 to investigate the effects of these Lactobacillus strains on liver injuries in NZB/W F1 mice. The results thus obtained reveal that supplementary GMNL-32, GMNL-89 or GMNL-263 in NZB/W F1 mice ameliorates hepatic apoptosis and inflammatory indicators, such as matrix metalloproteinase-9 activity and Creactive protein and inducible nitric oxide synthase expressions. In addition, supplementation with GMNL-32, GMNL-89 or GMNL-263 in NZB/ W F1 mice reduced the expressions of hepatic IL- $1 \beta$, IL- 6 and TNF- $\alpha$ proteins by suppressing the mitogen-activated protein kinase and NF- $\kappa$ B signalling pathways. These findings, presented here for the first time, reveal that GMNL-32, GMNL-89 and GMNL-263 mitigate hepatic inflammation and apoptosis in lupus-prone mice and may support an alternative remedy for liver disorders in cases of SLE.

Key words: Probiotics: Lactobacillus paracasei GMNL-32: Lactobacillus reuteri GMNL-89: Lactobacillus reuteri GMNL-263: Systemic lupus erythematosus: Liver disorders

Probiotics or probiotic bacteria generally refer to live microorganisms pre-existing in human bodies that are beneficial to intestinal tract health, or micro-organisms administered from external sources that favour any aspect of human health ${ }^{(1,2)}$. Various food products that are made from probiotics are regarded as functional foods ${ }^{(3,4)}$. Lactobacilli are being widely used in the development of novel bio-therapeutic probiotic formulations for managing various diseases. Indeed, the effects of probiotics on various diseases have been widely studied in both animal experiments and human clinical trials ${ }^{(5-8)}$. Evidence has demonstrated that Lactobacillus casei Shirota differentially the inflammatory cytokine responses of macrophages and T cells in either Peyer's patches or the spleen ${ }^{(9)}$. A recent study indicated that Lactobacillus jensenii TL2937 can interact with intestinal

Abbreviations: CRP, C-reactive protein; ERK, extracellular signal-regulated kinase; GMNL-32, Lactobacillus paracasei GMNL-32; GMNL-89, Lactobacillus reuteri GMNL-89; GMNL-263, Lactobacillus reuteri GMNL-263; IKK, IкB kinase; iNOS, inducible nitric oxide synthase; JNK, c-Jun N-terminal kinase; MAPK, mitogenactivated protein kinase; MMP, matrix metalloproteinase; SLE, systemic lupus erythematosus; TUNEL, terminal deoxynucleotidyl transferase dUTP nick end labelling.

* Corresponding authors: Dr B.-S. Tzang, fax +88642324 8195, email bstzang@csmu.edu.tw; Dr Y.-H. Chen, fax +886 65052152 , email ethan@genmont. com.tw 
epithelial cells and immune cells through Toll-like receptors (TLR) and improve animal and human health ${ }^{(10)}$. In another study, probiotic L. casei Zhang reduced the lipopolysaccharide/ D-galactosamine $\mathrm{N}$-induced expression of IL- $1 \beta$ and inducible nitric oxide synthase (iNOS) in the liver by modulating the TLR-mitogen-activated protein kinase (MAPK)-PPAR- $\gamma$ signalling pathways and intestinal microbiota in a rat model of acute liver failure ${ }^{(11)}$. These findings reveal that certain probiotics potentially have beneficial effects on both animals and humans by regulating the immune system.

Systemic lupus erythematosus (SLE) is an autoimmune inflammatory disorder that affects various organs, including the liver ${ }^{(12)}$. Notably, an increasing body of evidence reveals that liver abnormality is more common in cases of SLE than has typically been reported. Significantly increased liver abnormalities, such as elevated aspartate aminotransferase, alanine aminotransferase, fatty liver, portal inflammation, histopathological change and apoptosis, have been observed in the livers of SLE patients and lupus-prone mice, relative to controls ${ }^{(13-17)}$. Interestingly, the intake of dietary supplements has recently been demonstrated to be strongly associated with disease activity and intestinal microbiota in both lupus-prone animals and SLE patients ${ }^{(18,19)}$. Therefore, supplementation with certain probiotics could be beneficial to immune function and gastrointestinal health in cases of SLE.

Growing evidence reveals that a damaged intestinal barrier, also known as 'leaky gut', enhances interactions between intestinal bacteria and liver receptors, such as toll-like receptors, causing various hepatic disorders, such as liver cirrhosis, nonalcoholic fatty liver diseases and hepatic encephalopathy. However, the alteration of gut microbiota by administrating probiotics has been demonstrated to have a potentially beneficial effect on hepatic disorders ${ }^{(20,21)}$. Our recent study revealed that heat-killed Lactobacillus reuteri GMNL-263 (GMNL-263) reduces liver and heart fibrosis in hamsters on a high-fat diet by suppressing transforming growth factor- $\beta^{(22)}$. Both live and heatkilled GMNL-263 significantly reduce obesity-induced metabolic abnormalities by reducing insulin resistance and hepatic steatosis formation $^{(23)}$. However, no investigation of the functions of Lactobacillus paracasei GMNL-32 (GMNL-32) and L. reuteri GMNL-89 (GMNL-89) has been performed. As hepatic abnormality has been strongly associated with the pathogenesis of $\mathrm{SLE}^{(12)}$, this study investigates whether orally administering GMNL-32, GMNL-89 and GMNL-263 reduces hepatic inflammation and apoptosis in NZB/W F1 mice.

\section{Methods}

\section{Preparation of Lactobacillus strains}

GMNL-32, GMNL-89 and GMNL-263 were obtained from GenMont Biotech, Inc., and the doses of these Lactobacillus strains $\left(10^{9}\right.$ colony-forming units (CFU)/mouse per d) that were used in this study were based on our previous publications ${ }^{(22,24)}$. The catalogue numbers at the Bioresource Collection and Research Center in Taiwan are 'BCRC 910220', 'BCRC 910340' and 'BCRC 910452', respectively. Powders of three Lactobacillus strains were prepared in PBS for use in the oral gavage treatment of mice.

\section{Animals and treatments}

This study was approved by the Institutional Animal Care and Use Committee at Chung Shan Medical University (approval no. 847). A total of thirty-two female NZB/W F1 mice of 6 weeks of age were purchased from Jackson Laboratory and housed under the supervision of the Institutional Animal Care and Use Committee at Chung Shan Medical University, Taichung, Taiwan. All animals were kept in a $12 \mathrm{~h}$ light $-12 \mathrm{~h}$ dark cycle and ambient temperature was maintained at $25^{\circ} \mathrm{C}$. Animals had free access to water and standard laboratory chow (LabDiet 5001; PMI Nutrition International Inc.). Animal welfare was maintained and experimental procedures were performed according to the National Institutes of Health Guide for the Care and Use of Laboratory Animals. All animals at an age of 8 weeks were randomly divided into four groups (each of eight mice) - control, GMNL-32, GMNL-89 and GMNL-263 groups, which are treated with PBS, $10^{9} \mathrm{CFU} /$ mouse per d of GMNL-32, GMNL-89 and GMNL-263, respectively, by oral gavage. The experimental period was 12 weeks long and the mice were killed by asphyxiation using $\mathrm{CO}_{2}$ at an age of 20 weeks. Liver tissues were collected and stored at $-80^{\circ} \mathrm{C}$ until analysis.

\section{Gel zymography}

Matrix metalloproteinase (MMP)-2 and MMP-9 activities were analysed by gelatin zymography assays as previously described $^{(25)}$. Protein lysates amounting to $25 \mu \mathrm{g}$ from the liver tissue of NZB/W F1 mice were separated onto an $8 \%$ SDS-PAGE gel containing $0.1 \%$ gelatin. Gels were washed for $30 \mathrm{~min}$ in $2.5 \%$ Triton X-100 to remove SDS and then soaked in a reaction buffer containing $40 \mathrm{~mm}$ TRIS-HCl (pH 8.0), $10 \mathrm{~mm}^{\mathrm{CaCl}_{2}}$ and $0.02 \%$ $\mathrm{NaN}_{3}$ for $30 \mathrm{~min}$. The gels were incubated at $37^{\circ} \mathrm{C}$ for $24 \mathrm{~h}$ after transferring to a fresh reaction buffer. Gelatinolytic activity was visualised by staining the gels with $0.5 \%$ Coomassie Brilliant Blue R-250; they were then destained with methanol-acetic acid water, and relative MMP levels were quantified by a gel documentation and analysis system (Appraise; Beckman Coulter).

\section{Haematoxylin-eosin staining}

Haematoxylin-eosin (H-E) staining was performed as described elsewhere ${ }^{(26)}$. Liver samples from the animals were excised and soaked in formalin and covered with wax. The waxed tissue blocks were cut into 5 - $\mu$ m-thick sections and prepared by deparaffinisation and dehydration. The sections were passed through a series of graded alcohols (100, 95 and $75 \%$ ) for $15 \mathrm{~min}$ each and then dyed with haematoxylin. Photomicrographs were obtained using Zeiss Axiophot microscopes.

\section{Detection of terminal deoxynucleotidyl transferase dUTP nick end labelling-positive cells}

Apoptotic cells were detected with terminal deoxynucleotidyl transferase dUTP nick end labelling (TUNEL) assay (Roche Applied Science) as described elsewhere ${ }^{(27)}$. Under florescence (excitation wavelength of $460 \mathrm{~nm}$ and detection in the range of $515-565 \mathrm{~nm}$ ), TUNEL-positive nuclei with fragmented DNA 
were illuminated in bright green. To visualise the nuclei, the tissue sections were reacted with $0 \cdot 1 \mu \mathrm{g} / \mathrm{ml} \mathrm{4}$, 6-diamidino-2phenylindole, and the nuclei were detected and photographed at $454 \mathrm{~nm}$ using a Zeiss Axiophot microscope.

\section{Protein preparation and immunoblotting}

All procedures were performed at $4^{\circ} \mathrm{C}$ in a cold room as previously described ${ }^{(28,29)}$. The liver tissues obtained from NZB/W F1 mice were homogenised in $600 \mu \mathrm{l}$ PRO-PREP solution (iNtRON Biotech) by thirty strokes using a Dounce Homogenizer (Kontes Glass). The homogenates were centrifuged at $13000 \mathrm{rpm}$ for $10 \mathrm{~min}$ at $4^{\circ} \mathrm{C}$ and the supernatant was then stored at $-80^{\circ} \mathrm{C}$ until use. The protein content in the supernatant was measured according to the procedures of a Bio-Rad protein assay kit (Bio-Rad Laboratories, Inc.). Immunoblotting was performed as described in our previous study. In brief, protein samples were separated in 10 or $12.5 \%$ SDS-PAGE and electrophoretically transferred to a nitrocellulose membrane (Amersham Biosciences). After blocking with $5 \%$ non-fat dry milk in $1 \times$ PBS, antibodies against C-reactive protein (CRP), iNOS, IL- $1 \beta$, TNF- $\alpha$, IL-6, caspase-3, phosphorylated extracellular signal-regulated kinase (p-ERK), ERK, phosphorylated P38 (p-P38), P38, phosphorylated c-Jun N-terminal kinase (p-JNK), JNK, IкB kinase (IKK) (Santa Cruz Biotechnology), NF- $\kappa \mathrm{B}$ (Merck Millipore) and $\beta$-actin (Upstates) were diluted in PBS with $2.5 \%$ bovine serum albumin and incubated for $1.5 \mathrm{~h}$ with gentle agitation at room temperature. The membranes were washed twice with PBS-Tween for $1 \mathrm{~h}$ and secondary antibody conjugated with horseradish peroxidase (HRP) (Santa Cruz Biotechnology) was added. Pierce's SuperSignal West Dura HRP Detection Kit (Pierce Biotechnology Inc.) was used to detect antigen-antibody complexes. Quantified results were performed by densitometry (Appraise).

\section{Statistical analysis}

Sample size was calculated using free sample size calculating software G*Power version 3.1.9.2 (Franz, Universitat Kiel). With a power of $80 \%, 0.05$ level of statistical significance and an effect size of $0 \cdot 8$, sample size for each test was calculated to be eight. A total of thirty-two mice were randomly assigned into one of the four experimental groups. All values are expressed as means and standard deviations. The comparisons in gel zymography, TUNEL and immunoblot among groups were performed using GraphPad Prism 5 software (GraphPad Software, Inc.) by one-way ANOVA followed by Tukey's multiple comparisons test. $P<0.05$ was considered to indicate a statistically significant difference. The significant differences were stressed with symbols as shown in figures.

\section{Results}

\section{Supplementation with Lactobacillus strains reduces hepatic}

matrix metalloproteinase-9 activities and C-reactive protein and inducible nitric oxide synthase expressions in lupus-prone mice

To clarify the anti-inflammatory effects of various Lactobacillus strains on lupus-prone mice, the activity of MMP-9 and expressions of CRP and iNOS proteins in the livers of NZB/W F1 mice that were given a control, GMNL-32, GMNL-89 or a GMNL-263 diet were determined. Notably, significantly lower MMP-9:MMP-2 activity ratios were detected in the livers of NZB/W F1 mice that were given a GMNL-32, GMNL-89 or a GMNL-263 diet, respectively, than in the livers of those that were given the control diet (Fig. 1(a)). In addition, significantly lower expressions of CRP and iNOS proteins were observed in the livers of NZB/W F1 mice that were given a GMNL-32, GMNL-89 or a GMNL-263 diet, respectively, than in the livers of those that were given the control diet (Fig. 1(c)). Fig. 1(d) and (e) present quantitative results concerning CRP and iNOS levels, respectively, relative to the $\beta$-actin level.

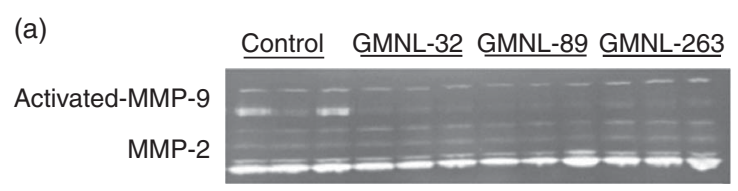

(b)
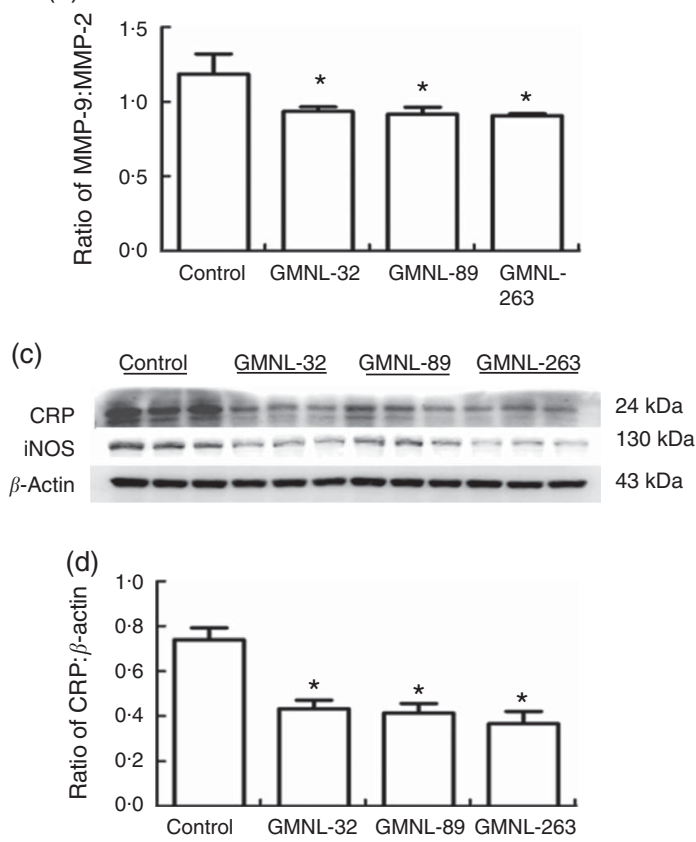

(e)

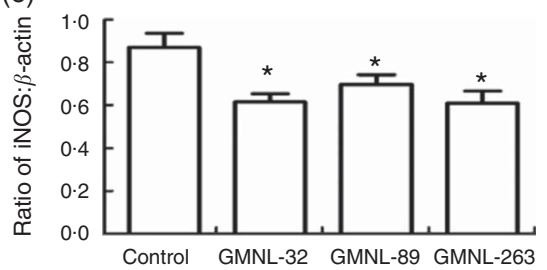

Fig. 1. Detection of matrix metalloproteinase (MMP)-9 activity and expressions of C-reactive protein (CRP) and inducible nitric oxide synthase (iNOS). Liver lysates were obtained from NZB/W F1 mice receiving control, Lactobacillus paracasei GMNL-32 (GMNL-32), Lactobacillus reuteri GMNL-89 (GMNL-89) or L. reuteri GMNL-263 (GMNL-263) diet, respectively. (a) Hepatic MMP-9 and MMP-2 activities. (b) Signal intensity of MMP-9 and MMP-2 activities were quantitated using a Phosphoimager and the ratio of MMP-9:MMP-2 is also presented. (c) Expression of CRP and iNOS proteins were detected by zymorgraphy and probed with antibodies against CRP and iNOS. Relative protein quantification of (d) CRP and (e) iNOS are represented by vertical bars on the basis of $\beta$-actin. Similar results were observed in three repeated experiments. Mean value was significantly different from that of the control group: ${ }^{*} P<0.05$. 
(a)

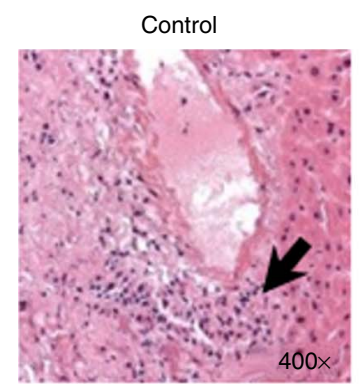

GMNL-32

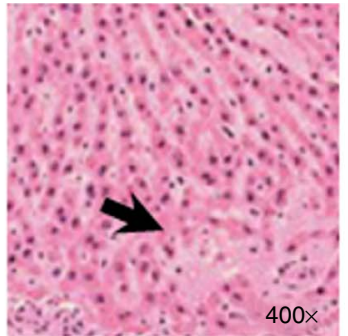

GMNL-89

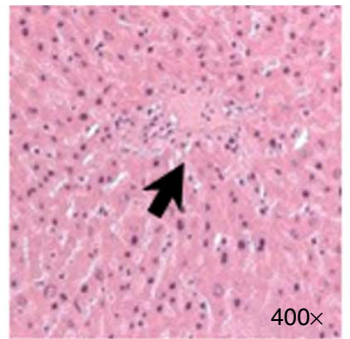

GMNL-263

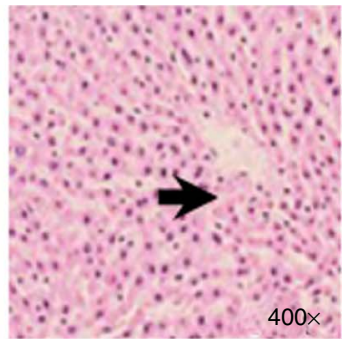

(b) Control

GMNL-32

GMNL-89

GMNL-263

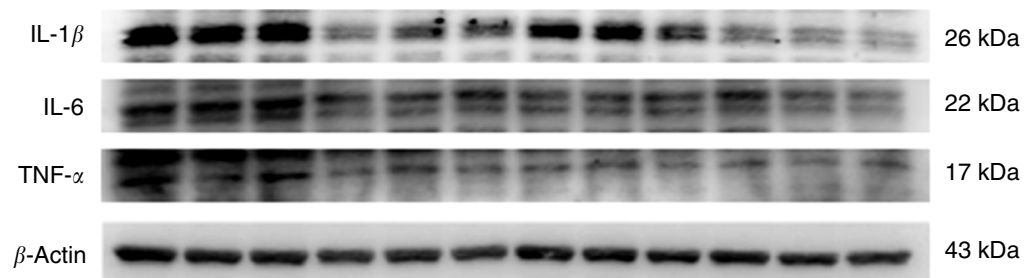

(c)

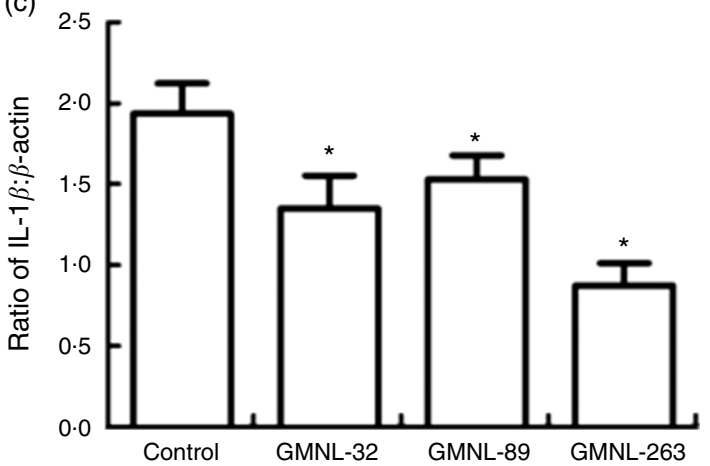

(d)

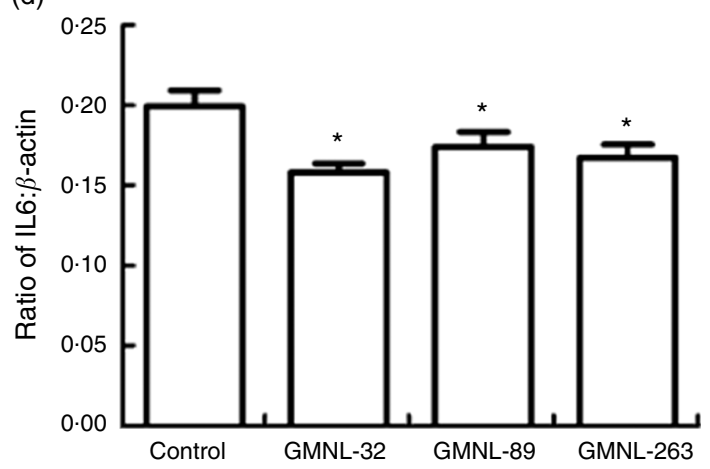

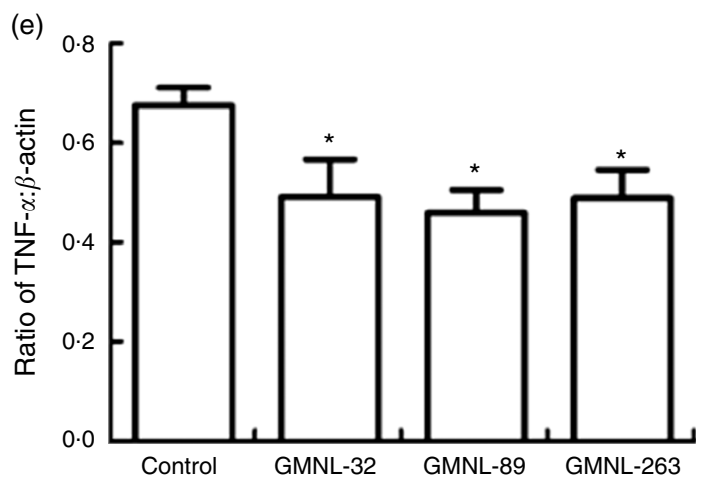

Fig. 2. Histopathological changes and expressions of IL-1 $\beta$, IL-6 and TNF- $a$. (a) Liver sections obtained from NZB/W F1 mice receiving control, Lactobacillus paracasei GMNL-32 (GMNL-32), Lactobacillus reuteri GMNL-89 (GMNL-89) or L. reuteri GMNL-263 (GMNL-263) diet, respectively, were stained with haematoxylineosin. Images of hepatic architecture were 400x magnified. Liver lysates obtained from NZB/W F1 mice receiving control, GMNL-32, GMNL-89 or GMNL-263 diet, respectively, were probed with antibodies against (b) IL-1 $\beta$, IL- 6 and TNF- $\alpha$. Relative protein quantification of (c) IL-1 $\beta$, (d) IL- 6 and (e) TNF- $a$ are represented by vertical bars on the basis of $\beta$-actin. Similar results were observed in three repeated experiments. Mean value was significantly different from that of the control group: ${ }^{*} P<0.05$.

Supplementation with Lactobacillus strains reduces hepatic lymphocyte infiltration and IL-1 $\beta, I L-6$ and TNF- $\alpha$ expressions in lupus-prone mice

To further investigate the effects of various Lactobacillus strains on hepatic histopathological changes and expressions of pro-inflammatory cytokines, IL- $1 \beta$, IL-6 and TNF- $\alpha$ were detected by $\mathrm{H}-\mathrm{E}$ staining and immunoblotting. Considerably greater lymphocyte infiltration was observed in the liver sections of NZB/W F1 mice that were given a GMNL-32, GMNL-89 or a GMNL-263 diet, respectively, than in the liver sections of mice that were given the control diet (Fig. 2(a)). Significantly lower 
(a)

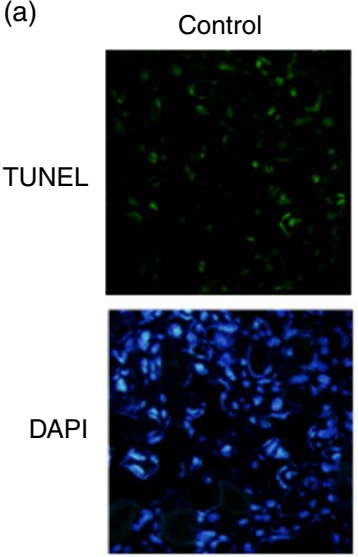

GMNL-32
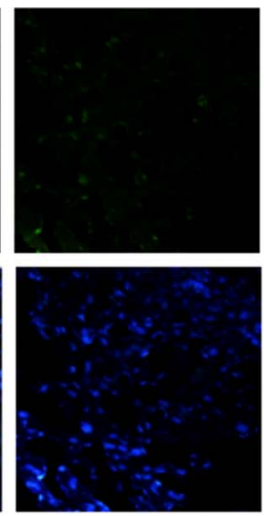

GMNL-89
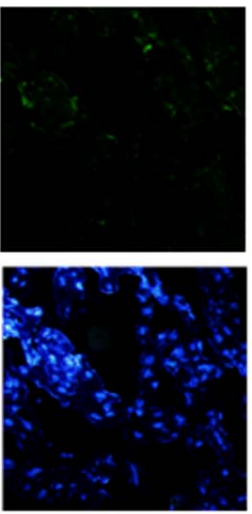

GMNL-263
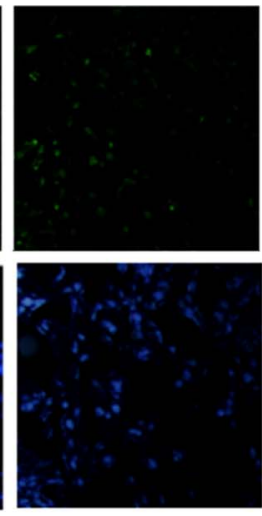

(b)

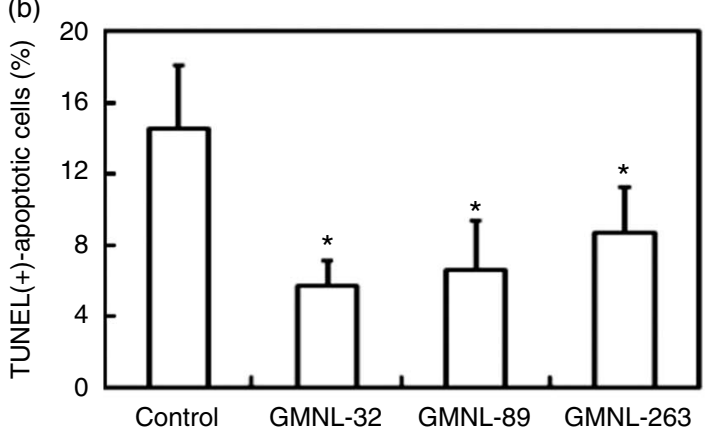

(c)

$$
\text { Control GMNL-32 GMNL-89 } \underline{\text { GMNL-263 }}
$$

Pro-caspase-3

Cleaved-caspase-3

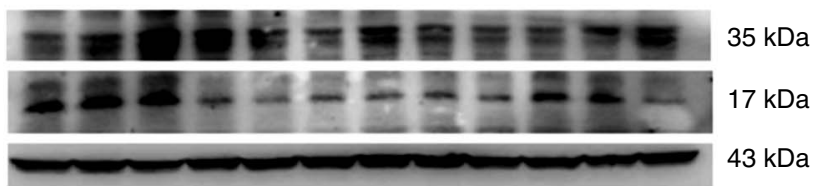

$\beta$-Actin

(d)

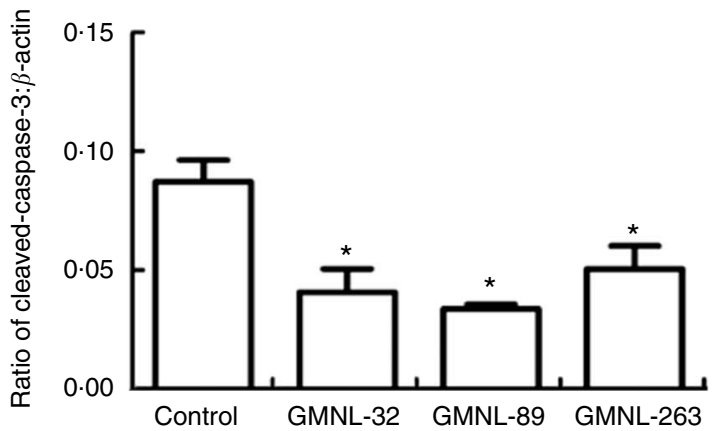

Fig. 3. Detection of terminal deoxynucleotidyl transferase dUTP nick end labelling (TUNEL)-positive cells and expression of caspase-3. Liver sections and liver lysates were obtained from NZB/W F1 mice receiving control, Lactobacillus paracasei GMNL-32 (GMNL-32), Lactobacillus reuteri GMNL-89 (GMNL-89) or L. reuteri GMNL-263 (GMNL-263) diet, respectively. (a) TUNEL assay was performed with liver sections. Fluorescein isothiocyanate (FITC)-labelled terminal deoxytransferase was bound to nicked end of DNA. 4, 6-diamidino-2-phenylindole (DAPI) staining was used as control. (b) The percentage of TUNEL-positive cells in liver sections are represented by vertical bars. (c) Expression of caspase-3 was detected with antibodies against caspase-3. Relative protein quantification of (d) cleaved caspase-3 are represented by vertical bars on the basis of $\beta$-actin. Similar results were observed in three repeated experiments. Mean value was significantly different from that of the control group: ${ }^{*} P<0.05$.

expressions of IL- $1 \beta$, IL- 6 and TNF- $\alpha$ proteins were detected in the livers of NZB/W F1 mice that were given a GMNL-32, GMNL-89 or GMNL-263 diet than in the livers of mice that were given the control diet (Fig. 2(b)). Fig. 2(c)-(e) present quantitative results concerning IL- $1 \beta$, IL- 6 and TNF- $\alpha$ levels, respectively, relative to $\beta$-actin level. 
(a)

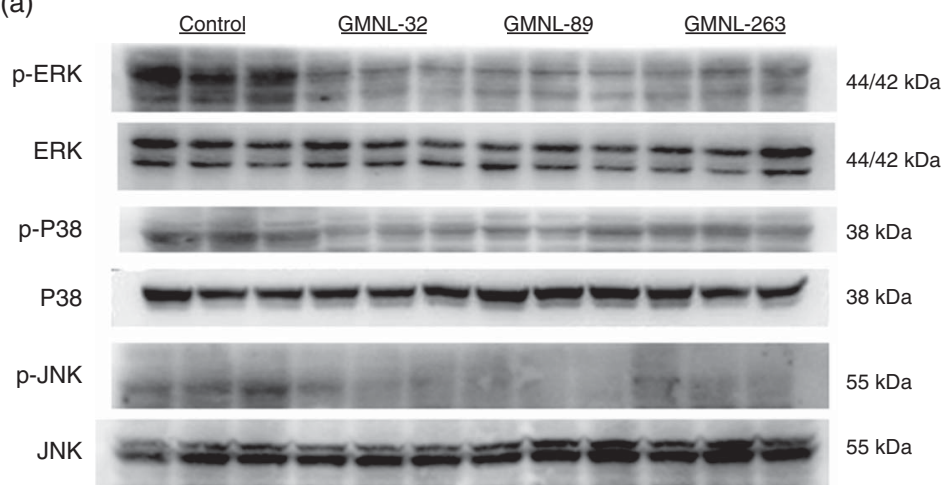

(b)

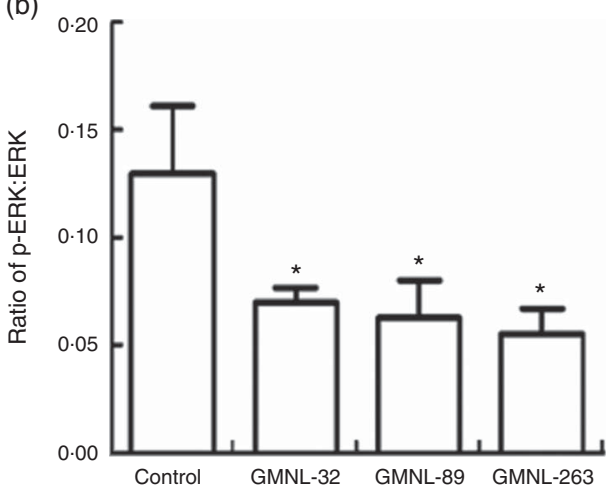

(c)

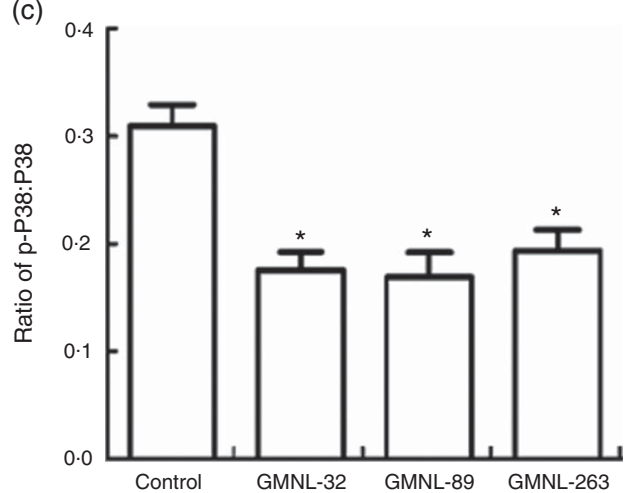

(d)

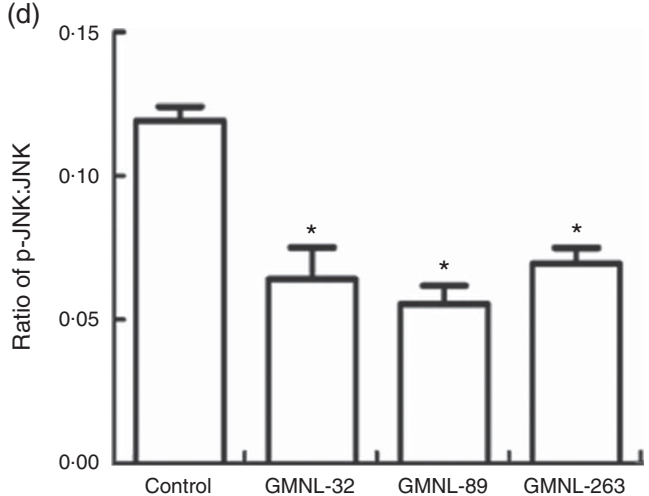

Fig. 4. Expression of extracellular signal-regulated kinase (ERK), P38 and c-Jun N-terminal kinase (JNK). Liver lysates obtained from NZB/W F1 mice receiving control, Lactobacillus paracasei GMNL-32 (GMNL-32), Lactobacillus reuteri GMNL-89 (GMNL-89) or L. reuteri GMNL-263 (GMNL-263) diet, respectively, were probed with antibodies against (a) ERK, phosphorylated ERK (p-ERK), P38, phosphorylated P38 (p-P38), JNK and phosphorylated JNK (p-JNK). Relative protein quantification of (b) p-ERK, (c) p-P38 and (d) p-JNK are represented by vertical bars on the basis of ERK, P38 and JNK, respectively. Similar results were observed in three repeated experiments. Mean value was significantly different from that of the control group: ${ }^{*} P<0.05$.

\section{Supplementation with Lactobacillus strains reduces hepatic apoptosis in lupus-prone mice}

To investigate the effects of various Lactobacillus strains on hepatic apoptosis, TUNEL-positive cells and cleaved caspase-3 protein were detected. Significantly fewer TUNEL-positive cells were detected in the livers of NZB/W F1 mice that were given the GMNL-32, GMNL-89 or the GMNL-263 diet, than in those of mice that were given the control diet (Fig. 3(a)). Fig. 3(b) presents the percentage of cells that were TUNEL-positive. Significantly less cleaved caspase- 3 protein was detected in the livers of NZB/W F1 mice that were given the GMNL-32,
GMNL-89 or GMNL-263 diet, respectively, than in the livers of mice that were given the control diet (Fig. 3(c)). Fig. 3(d) presents quantitative results concerning the cleaved caspase- 3 level relative to the $\beta$-actin level.

Signalling molecules involved in reduction of hepatic injury in lupus-prone mice by Lactobacillus strains

To identify the signalling molecules that may be involved in the reduction of liver injury in lupus-prone mice by Lactobacillus strains, the signalling molecules of the MAPK and NF- $\kappa$ B pathways were examined. Significantly lower ratios of p-ERK:ERK, 
(a)

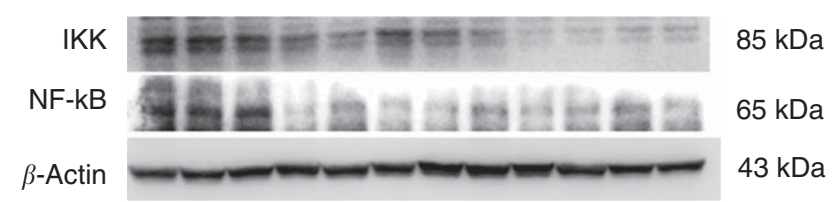

(b)

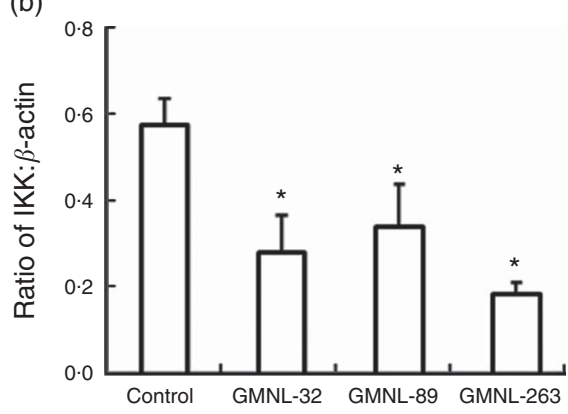

(c)

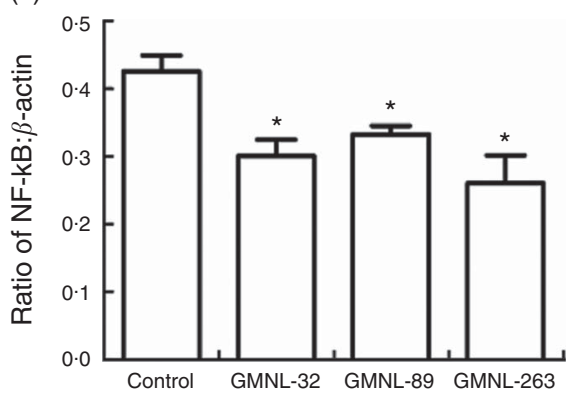

Fig. 5. Expression of $\mathrm{I} \mathrm{kB}$ kinase (IKK) and NF-kB. Liver lysates obtained from NZB/W F1 mice receiving control, Lactobacillus paracasei GMNL-32 (GMNL-32), Lactobacillus reuteri GMNL-89 (GMNL-89) or L. reuteri GMNL-263 (GMNL-263) diet, respectively, were probed with antibodies against (a) IKK and $\mathrm{NF}-\mathrm{KB}$. Relative protein quantification of (b) IKK and (c) NF-KB are represented by vertical bars on the basis of $\beta$-actin. Similar results were observed in three repeated experiments. Mean value was significantly different from that of the control group: * $P<0.05$.

p-P38:P38 and p-JNK:JNK were observed in the livers of NZB/W F1 mice that had been given a GMNL-32, GMNL-89 or a GMNL-263 diet, respectively, than in the livers of mice that had been given the control diet (Fig. 4(a)). Fig. 4(b)-(d) present the ratios of $\mathrm{p}$-ERK:ERK, $\mathrm{p}-\mathrm{P} 38: \mathrm{P} 38$ and $\mathrm{p}-\mathrm{JNK}: \mathrm{JNK}$, respectively. The expressions of both IKK and $\mathrm{NF}-\kappa \mathrm{B}$ proteins were significantly lower in the livers of NZB/WW F1 mice that had been given a GMNL-32, GMNL-89 or a GMNL-263 diet, respectively, than in the livers of mice that had been given the control diet (Fig. 5(a)). Fig. 5(b) and (c) present quantitative results concerning IKK and $\mathrm{NF}-\kappa \mathrm{B}$ levels, respectively, relative to the $\beta$-actin level.

\section{Discussion}

Certain probiotics are known to exhibit diverse functions, such as modulation of the immune system and homoeostasis of intestinal microbiota against various diseases ${ }^{(1,2)}$. This study is the first to reveal that oral gavage of Lactobacillus strains, including GMNL-32, GMNL-89 and GMNL-263, mitigates hepatic apoptosis and inflammatory indicators, such as MMP-9 activity and CRP and iNOS expressions in lupus-prone mice. Supplementation with GMNL-32, GMNL-89 or GMNL-263 in NZB/W F1 mice also reduces the expressions of hepatic IL- $1 \beta$, IL- 6 and TNF- $\alpha$ proteins by reducing MAPK/NF- $\kappa$ B inflammatory signalling. These findings reveal the beneficial effects of GMNL-32, GMNL-89 and GMNL-263 on hepatic inflammation and apoptosis in lupus-prone mice and suggest an alternative treatment for liver disorders in cases of SLE.

Although microbes and diet have been suggested to play critical roles in initiating autoimmunity ${ }^{(18,19,30)}$, the effect of gut microflora on the initiation and progression of SLE remains unclear. Gastrointestinal symptoms are common in SLE patients, and more than half of them are caused by adverse reactions to medications and microbial infections ${ }^{(30)}$. These findings suggest that an imbalance of gastrointestinal microflora is involved in the pathogenesis of SLE. Recent studies have paved the way to treatments that involve probiotics ${ }^{(2,31)}$. Evidence has shown that certain Lactobacillus strains can promote the production of anti-inflammatory cytokines such as IL-10 and TNF- $\alpha$, and thus can be used to treat autoimmune diseases such as $\operatorname{SLE}^{(2,32,33)}$. However, few studies have investigated whether Lactobacillus is effective in treating liver injuries that are associated with autoimmune diseases such as SLE. This study reveals that supplementation with GMNL-32, GMNL-89 or GMNL-263 mitigates hepatic apoptosis and inflammation in NZB/W F1 mice by reducing MMP-9 activity and the expressions of CRP and iNOS proteins. Furthermore, supplementation with GMNL-32, GMNL-89 or GMNL-263 reduced the expressions of inflammatory cytokines IL- $1 \beta$, IL- 6 and TNF- $\alpha$ in the livers of NZB/W F1 mice. These results suggest that GMNL-32, GMNL-89 and GMNL-263 have beneficial effects in the treatment of hepatic inflammation and apoptosis in cases of SLE.

The major output of MAPK signalling downstream of inflammatory cytokines is a pro-inflammatory response. The TNF superfamily ligands subsequently cause a cascade and then phosphorylate JNK and P38. Activated JNK and P38 translocate to the nucleus and activate multiple transcription factors such as NF- $\kappa \mathrm{B}$, which leads to the expression of proinflammatory cytokines ${ }^{(34)}$. Indeed, special attention has been paid to the specific role of the autoantibody-induced activation of P38 MAPK-mediated immunopathology in the pathogenesis of autoimmune diseases ${ }^{(35)}$. SLE is a highly complex autoimmune disorder with unknown aetiology in which a diffuse, chronic inflammatory reaction plays an important aetiological role ${ }^{(12,36)}$. Clinical trials in SLE are focusing on the development of such agents as belimumab to control the activation of T- and B-lymphocytes by targeting the B-cell survival factor BAFF (Bcell activating factor ${ }^{(36)}$. However, the efficacy of belimumab is limited. Recently, emphasis has been placed on elucidating the association between inflammasome and pro-inflammatory cell death (pyroptosis), which results in the modification of autoantigens and the generation of autoimmunity in lupus ${ }^{(37)}$. Therefore, the potential effectiveness of the modulation of MAPK pathways in treating autoimmune and inflammatory diseases such as SLE should be considered ${ }^{(36)}$. Notably, this study is the first to identify the reduction of hepatic IL- $1 \beta$, 


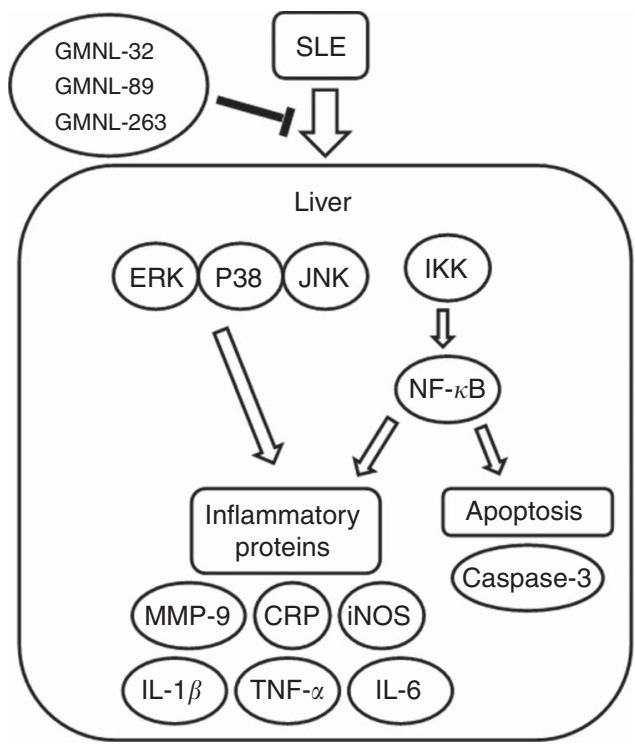

Fig. 6. Schematic illustration of the possible mechanism of Lactobacillus paracasei GMNL-32 (GMNL-32), Lactobacillus reuteri GMNL-89 (GMNL-89) and $L$. reuteri GMNL-263 (GMNL-263) involved in systemic lupus erythematosus (SLE)-associated liver injuries. Lactobacillus strains of GMNL-32, GMNL-89 and GMNL-263 ameliorate hepatic apoptosis and inflammation through mitogenactivated protein kinase/NF-KB signalling in NZB/W F1 mice. ERK, extracellular signal-regulated kinase; JNK, c-Jun N-terminal kinase; IKK, IKB kinase; MMP, matrix metalloproteinase; iNOS, inducible nitric oxide synthase.

IL- 6 and TNF- $\alpha$ protein expression through reducting the MAPK/NF- $\kappa \mathrm{B}$ signalling. Although the interplay of GMNL-32, GMNL-89 and GMNL-263 with the gastrointestinal microbiota and immune cells requires further investigation, this study suggests that certain probiotics mitigate hepatic inflammation in cases of SLE.

\section{Conclusions}

Although the precise mechanism by which GMNL-32, GMNL-89 and GMNL-263 act on liver injuries in cases of SLE requires further investigation, we postulate that the anti-apoptosis and anti-inflammation effects of GMNL-32, GMNL-89 and GMNL-263 probably involve their regulatory effects on gastrointestinal microbiota, intestinal epithelial cells and immune cells in gutassociated lymphoid tissue. As displayed in Fig. 6, GMNL-32, GMNL-89 and GMNL-263 significantly mitigate liver apoptosis in NZB/W F1 mice by reducing IKK/NF- $\kappa \mathrm{B}$ signalling. GMNL-32, GMNL-89 and GMNL-263 also significantly mitigate liver inflammation by reducing the activity of MMP-9 and the expressions of CRP, iNOS, IL- $1 \beta$, IL- 6 and TNF- $\alpha$ proteins by inhibiting MAPK/NF- $\kappa \mathrm{B}$ inflammatory signalling. These findings reveal the inhibition of liver injuries in lupus-prone mice by GMNL-32, GMNL-89 and GMNL-263, and suggest the therapeutic potential of GMNL-32, GMNL-89 and GMNL-263 against hepatic injuries in SLE.

\section{Acknowledgements}

This work was supported by GenMont Biotech, Inc., Tainan, Taiwan (E0150100). GenMont Biotech had no role in the design, analysis or writing of this article.
T.-C. H., C.-Y. H., Y.-H. C. and B.-S. T. conceived and designed the experiments; T.-C. H., C.-Y. H., C.-H. L., K.-C. H., Y.-H. C. and B.-S. T. performed the experiments and analysed the data; and T.-C. H., Y.-H. C. and B.-S. T. wrote the paper.

None of the authors has any conflicts of interest to declare.

\section{References}

1. Gerritsen J, Smidt H, Rijkers GT, et al. (2011) Intestinal microbiota in human health and disease: the impact of probiotics. Genes Nutr 6, 209-240.

2. Dwivedi M, Kumar P, Laddha NC, et al. (2016) Induction of regulatory $\mathrm{T}$ cells: a role for probiotics and prebiotics to suppress autoimmunity. Autoimmun Rev 15, 379-392.

3. Nagpal R, Kumar A, Kumar M, et al. (2012) Probiotics, their health benefits and applications for developing healthier foods: a review. FEMS Microbiol Lett 334, 1-15.

4. Govender M, Choonara YE, Kumar P, et al. (2014) A review of the advancements in probiotic delivery: conventional vs. nonconventional formulations for intestinal flora supplementation. AAPS PharmSciTech 15, 29-43.

5. Matsuzaki T, Nagata Y, Kado S, et al. (1997) Effect of oral administration of Lactobacillus casei on alloxan-induced diabetes in mice. APMIS 105, 637-642.

6. Kaur IP, Chopra K \& Saini A (2002) Probiotics: potential pharmaceutical applications. Eur J Pharm Sci 15, 1-9.

7. de Moreno de LeBlanc A, Matar C \& Perdigon G (2007) The application of probiotics in cancer. Br J Nutr 98, Suppl. 1, S105-S110.

8. Laitinen K, Poussa T, Isolauri E, et al. (2009) Probiotics and dietary counselling contribute to glucose regulation during and after pregnancy: a randomised controlled trial. Br J Nutr 101, 1679-1687.

9. Shida K, Nanno M \& Nagata S (2011) Flexible cytokine production by macrophages and $\mathrm{T}$ cells in response to probiotic bacteria: a possible mechanism by which probiotics exert multifunctional immune regulatory activities. Gut Microbes 2, 109-114.

10. Villena J \& Kitazawa H (2014) Modulation of intestinal TLR4inflammatory signaling pathways by probiotic microorganisms: lessons learned from Lactobacillus jensenii TL2937. Front Immunol 4, 512.

11. Wang Y, Xie J, Li Y, et al. (2016) Probiotic Lactobacillus casei Zhang reduces pro-inflammatory cytokine production and hepatic inflammation in a rat model of acute liver failure. Eur J Nutr 55, 821-831.

12. Hahn BH (1993) An overview of the pathogenesis of systemic lupus erythematosus. In Dubois' Lupus Erythematosus, pp. 69-76 [DJ Wallace and BH Hahn, editors]. Philadelphia, PA: Williams and Wilkins.

13. Abraham S, Begum S \& Isenberg D (2004) Hepatic manifestations of autoimmune rheumatic diseases. Ann Rheum Dis 63, 123-129.

14. Lu MC, Li KJ, Hsieh SC, et al. (2006) Lupus-related advanced liver involvement as the initial presentation of systemic lupus erythematosus. J Microbiol Immunol Infect 39, 471-475.

15. Grover S, Rastogi A, Singh J, et al. (2014) Spectrum of histomorphologic findings in liver in patients with SLE: a review. Hepat Res Treat 2014, 562979.

16. Hsu TC, Huang CY, Chiang SY, et al. (2008) Transglutaminase inhibitor cystamine alleviates the abnormality in liver from NZB/W F1 mice. Eur J Pharmacol 579, 382-389.

17. Tzang BS, Chiang SY, Lai WX, et al. (2008) Treatment with cystamine reduces apoptosis in liver from NZB/W F1 mice. Int Immunopharmacol 8, 589-596. 
18. Cuervo A, Hevia A, López P, et al. (2015) Association of polyphenols from oranges and apples with specific intestinal microorganisms in systemic lupus erythematosus patients. Nutrients 7, 1301-1317.

19. Johnson BM, Gaudreau MC, Al-Gadban MM, et al. (2015) Impact of dietary deviation on disease progression and gut microbiome composition in lupus-prone SNF1 mice. Clin Exp Immunol 181, 323-337.

20. Lo RS, Austin AS \& Freeman JG. (2014) Is there a role for probiotics in liver disease? ScientificWorldJournal 2014, 874768.

21. Paolella G, Mandato C, Pierri L, et al. (2014) Gut-liver axis and probiotics: their role in non-alcoholic fatty liver disease. World J Gastroenterol 20, 15518-15531.

22. Ting WJ, Kuo WW, Hsieh DJ, et al. (2015) Heat killed Lactobacillus reuteri GMNL-263 reduces fibrosis effects on the liver and heart in high fat diet-hamsters via TGF- $\beta$ suppression. Int J Mol Sci 16, 25881-25896.

23. Hsieh FC, Lan CC, Huang TY, et al. (2016) Heat-killed and live Lactobacillus reuteri GMNL-263 exhibit similar effects on improving metabolic functions in high-fat diet-induced obese rats. Food Funct 7, 2374-2388.

24. Liao PH, Kuo WW, Kuo CH, et al. (2016) Lactobacillus reuteri GMNL-263 reduces hyperlipidaemia and the heart failure process in high-calorie diet-fed induced heart dysfunction in rats. J Funct Foods 20, 226-235.

25. Hsu TC, Chiu CC, Chang SC, et al. (2016) Human parvovirus B19 VP1u Protein as inflammatory mediators induces liver injury in naïve mice. Virulence 7, 110-118.

26. Lin CJ, Chiu CC, Chen YC, et al. (2015) Taurine attenuates hepatic inflammation in chronic alcohol-fed rats through inhibition of TLR4/MyD88 signaling. J Med Food 18, 1291-1298.
27. Hsu TC, Chiu CC, Lin HL, et al. (2015) Attenuated effects of deep-sea water on hepatic apoptosis in STZ-induced diabetic rats. Chin J Physiol 58, 197-205.

28. Tzang BS, Lin TM, Tsai CC, et al. (2011) Increased cardiac injury in NZB/W F1 mice received antibody against human parvovirus B19 VP1 unique region protein. Mol Immunol 48, 1518-1524.

29. Chiu CC, Shi YF, Yang JJ, et al. (2014) Effects of human Parvovirus B19 and Bocavirus VP1 unique region on tight junction of human airway epithelial A549 cells. PLOS ONE 9, e107970.

30. Tian XP \& Zhang X (2010) Gastrointestinal involvement in systemic lupus erythematosus: insight into pathogenesis, diagnosis and treatment. World $J$ Gastroenterol 16, 2971-2977.

31. Dongarrà ML, Rizzello V, Muccio L, et al. (2013) Mucosal immunology and probiotics. Curr Allergy Asthma Rep 13, $19-26$.

32. Honad K \& Littman DR (2012) The microbiome in infectious disease and inflammation. Ann Rev Immunol 30, 758-795.

33. Lescheid DW (2014) Probiotics as regulators of inflammation: a review. Funct Foods Health Dis 4, 299-311.

34. Arthur JS \& Ley SC (2013) Mitogen-activated protein kinases in innate immunity. Nat Rev Immunol 13, 679-692.

35. Mavropoulos A, Orfanidou T, Liaskos C, et al. (2013) p38 MAPK signaling in pemphigus: implications for skin autoimmunity. Autoimmune Dis 2013, 728529.

36. Gottschalk TA, Tsantikos E \& Hibbs ML (2015) Pathogenic inflammation and its therapeutic targeting in systemic lupus erythematosus. Front Immunol 6, 550.

37. Buyon JP, Cohen P, Merrill JT, et al. (2015) A highlight from the LUPUS 2014 meeting: eight great ideas. Lupus Sci Med $\mathbf{2}$, e000087. 